\title{
Star-product functions in higher-spin theory and locality
}

\author{
M.A. Vasiliev \\ I.E. Tamm Department of Theoretical Physics, Lebedev Physical Institute, \\ Leninsky prospect 53, 119991, Moscow, Russia \\ E-mail: vasiliev@lpi.ru
}

ABSTRACT: Properties of the functional classes of star-product elements associated with higher-spin gauge fields and gauge parameters are elaborated. Cohomological interpretation of the nonlinear higher-spin equations is given. An algebra $\mathcal{H}$, where solutions of the nonlinear higher-spin equations are valued, is found. A conjecture on the classes of starproduct functions underlying (non)local maps and gauge transformations in the nonlinear higher-spin theory is proposed.

KeYwords: Higher Spin Gravity, Higher Spin Symmetry

ARXIV EPRINT: 1502.02271

To my mother 


\section{Contents}

1 Introduction $\quad 1$

2 HS star product $\quad 2$

3 HS equations in $\boldsymbol{A d S}_{\mathbf{4}} \quad 4$

3.1 Nonlinear system and its cohomological interpretation 4

$\begin{array}{ll}3.2 & \text { Perturbative analysis }\end{array}$

4 Functional spaces $\quad 7$

$\begin{array}{lll}4.1 & \text { Algebra } V_{0,0} & 7\end{array}$

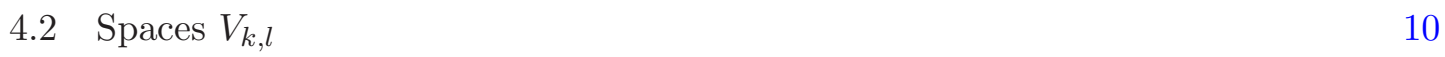

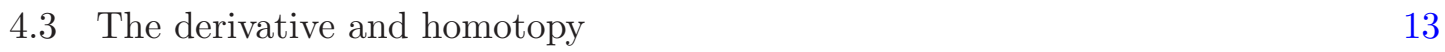

$\begin{array}{lll}4.4 & \text { Inner and boundary spaces } & 13\end{array}$

5 HS field algebra $\mathcal{H}$ and local algebra $\mathcal{H}^{\text {loc }} \quad \mathbf{1 4}$

$\begin{array}{lll}5.1 \text { HS field algebra } & 14\end{array}$

$\begin{array}{lll}5.2 & \text { Local HS algebra } & 15\end{array}$

6 Locality conjecture $\quad 16$

$\begin{array}{llr}7 & \text { Conclusion } & 18\end{array}$

A Proof of Lemma $5 \quad 20$

$\begin{array}{ll}\text { B Local HS algebra and Weyl star product } & 21\end{array}$

\section{Introduction}

In this paper we identify functional classes of star-product elements relevant to the analysis of nonlinear higher-spin (HS) equations. Our results give the cohomological interpretation of the terms responsible for interactions and shed light on the important issue of (non)locality in HS theories leading to a conjecture on the classes of (non)local functionals, field redefinitions and gauge transformations. This identification also restricts possible generalizations of the HS equations.

Importance of the issue of locality in HS theory was realized in [1] where it was shown that, by a seemingly local field redefinition induced by the so-called integrating flow found in the same paper for the $3 d$ HS theory, it is possible to get rid of currents from the r.h.s. of HS field equations including the stress tensor in the spin-two sector. It was argued in [1] that this result implies that the field transformation induced by the integrating flow 
is nonlocal. In [2], where this issue was further elaborated in terms of currents, such transformations were called pseudolocal. In the AdS background they have a form of an infinite derivative expansion

$$
\phi \rightarrow \phi^{\prime}=\phi+\sum_{n} a_{n m}(\rho D)^{n} \phi(\rho D)^{m} \phi+\ldots,
$$

where $\rho$ is the AdS radius and $D$ is the space-time covariant derivative. The problem is to find restrictions on the coefficients $a_{n m}$ distinguishing between truly non-local and generalized local field redefinitions which may contain an infinite number of terms but the coefficients $a_{n m}$ decrease fast enough with $n$ and $m$. Note that the problems in $A d S_{d}$ and Minkowski space are essentially different since the expansion (1.1) does not make sense in the naive limit $\rho \rightarrow \infty$.

In the unfolded form of the HS theories suggested in $[1,3,4]$ the space-time dependence is encoded in additional twistor-like variables $Z^{A}$ and $Y^{A}$ where the meaning of the index $A$ depends on the model. In the twistor-like variables, (1.1) is substituted by

$$
\phi \rightarrow \phi^{\prime}=\phi+\sum_{n m k l} b_{n m k l}\left(\left(\frac{\partial}{\partial Z}\right)^{n}\left(\frac{\partial}{\partial Y}\right)^{m} \phi\right)\left(\left(\frac{\partial}{\partial Z}\right)^{k}\left(\frac{\partial}{\partial Y}\right)^{l} \phi\right)+\ldots
$$

and the problem is to find restrictions on the coefficients $b_{n m k l}$. Being reformulated in terms of appropriate classes of functions, this is one of the goals of this paper. Specifically, we identify such restrictions on the maps between star-product elements in HS theory, that are algebraically consistent (form an algebra) and rule out the nonlocal transformations resulting from the integrating flow of [1] and similar. These will be conjectured to represent local maps in the HS theory. The class of allowed gauge transformations is also identified.

The rest of the paper is organized as follows. In section 2 we recall properties of the HS star product underlying nonlinear HS theories. In section 3 the structure of the field equations for the nonlinear $A d S_{4}$ HS theory is recalled and their cohomological interpretation is discussed. In section 4 functional spaces of star product elements underlying our construction are introduced. The algebra $\mathcal{H}$ where HS fields are valued is defined in section 5 . The locality conjecture is formulated in section 6 . Conclusions and perspectives are discussed in section 7. Details of the proof of Lemma 5 are presented in appendix A. A relation between the Weyl star product and the algebra $\mathcal{H}$ is briefly discussed in appendix B.

\section{HS star product}

HS equations were formulated in $[1,3,4]$ in terms of the associative $H S$ star product $*$ which acts on functions of two variables $Z_{A}$ and $Y_{A}$

$$
(f * g)(Z ; Y)=\frac{1}{(2 \pi)^{M}} \int d^{M} U d^{M} V \exp \left[i U^{A} V^{B} C_{A B}\right] f(Z+U ; Y+U) g(Z-V ; Y+V),
$$

where $C_{A B}=-C_{B A}(A, B, \ldots=1,2, \ldots, M)$ is nondegenerate allowing to raise and lower indices

$$
Y^{A}=C^{A B} Y_{B}, \quad Y_{A}=Y^{B} C_{B A},
$$


and $U^{A}, V^{B}$ are real integration variables. Star product (2.1), normalized so that 1 is its unit element, i.e., $f * 1=1 * f=f$, yields a specific realization of the Weyl algebra ${ }^{1}$

$$
\left[Y_{A}, Y_{B}\right]_{*}=-\left[Z_{A}, Z_{B}\right]_{*}=2 i C_{A B}, \quad\left[Y_{A}, Z_{B}\right]_{*}=0, \quad[a, b]_{*}=a * b-b * a
$$

and possesses a supertrace operation

$$
\operatorname{str}(f(Z ; Y))=\frac{1}{(2 \pi)^{M}} \int d^{M} U d^{M} V \exp \left[-i U^{A} V^{B} C_{A B}\right] f(U ; V)
$$

obeying the cyclic property

$$
\operatorname{str}(f * g)=\operatorname{str}(g * f)
$$

provided that the coefficients of the expansions of $f(Z ; Y)$ and $g(Z ; Y)$ in powers of $Z_{A}$ and $Y_{A}$ are (anti)commuting for $f(Z ; Y)$ and $g(Z ; Y)$ (odd)even under $f(-Z ;-Y)=$ $(-1)^{\pi_{f}} f(Z ; Y)$.

An important property of star product (2.1) is that it admits the inner Klein operator

$$
\Upsilon=\exp i Z_{A} Y^{A}
$$

which obeys

$$
\begin{aligned}
\Upsilon * \Upsilon & =1, \\
\Upsilon * f(Z ; Y) & =f(-Z ;-Y) * \Upsilon .
\end{aligned}
$$

The Klein operator $\Upsilon_{Y}$ for the star product of $Z$-independent functions, which amounts to the Weyl star product

$$
(f * g)(Y)=\frac{1}{(2 \pi)^{M}} \int d^{M} U d^{M} V \exp \left[i U^{A} V^{B} C_{A B}\right] f(Y+U) g(Y+V),
$$

is the $\delta$-function [5]

$$
\Upsilon_{Y}=(2 \pi)^{\frac{M}{2}} \delta^{M}(Y)
$$

Indeed, from (2.9) it follows that

$$
\delta^{M}(Y) * f(Y)=f(-Y) * \delta^{M}(Y) .
$$

$\Upsilon_{Y}$ squares to unity

$$
\Upsilon_{Y} * \Upsilon_{Y}=1 .
$$

(For $\hbar \neq 1$ reinserted into the definition of the star product, the r.h.s. of this relation is proportional to $\hbar^{-M}$ and, as anticipated, becomes infinite in the classical limit.)

An important property of the Klein operator is that it generates the Fourier transform:

$$
f(Y) * \Upsilon_{Y}=\tilde{f}(Y):=\frac{1}{(2 \pi)^{\frac{M}{2}}} \int d^{M} U \exp \left[-i U_{A} Y^{A}\right] f(U)
$$

\footnotetext{
${ }^{1}$ Weyl algebra is the algebra of oscillators. It should not be confused with the Weyl star product describing the product law in a specific frame of the Weyl algebra.
} 
For $Z$-independent elements $f(Z ; Y)=f(Y)(2.4)$ gives the well-known result [6]

$$
\operatorname{str}(f(Y))=f(0) .
$$

From here it follows that though $\Upsilon_{Y}$ is well behaving with respect to the star product its supertrace is divergent

$$
\operatorname{str}\left(\Upsilon_{Y}\right)=\infty \sim \delta^{M}(0) .
$$

Analogously, one can define

$$
\Upsilon_{Z}=(2 \pi)^{\frac{M}{2}} \delta^{M}(Z)
$$

Klein operator (2.6) results from the star product (2.1) of the Klein operators in the $Y$ and $Z$-sectors

$$
\Upsilon=\Upsilon_{Y} * \Upsilon_{Z}
$$

From (2.14) it follows that in the Weyl star product $\star$, which is the direct product of the Weyl star products in the $Y$ and $Z$ sectors,

$$
\operatorname{str}\left(\Upsilon_{Y} \star \Upsilon_{Z}\right)=\operatorname{str}\left(\Upsilon_{Y}\right) \operatorname{str}\left(\Upsilon_{Z}\right) \sim \delta^{2 M}(0)
$$

On the other hand, the supertrace is insensitive to a basis of the Weyl algebra, i.e., to the form of the star product. Hence $\operatorname{str}(\Upsilon)$ remains divergent as $\delta^{2 M}(0)$ in the HS star product. Analogously $\operatorname{str}(f)=\infty$ for any $f(Z ; Y)$ behaving in $Y$ and $Z$ like $\Upsilon$. This fact plays the key róle in [7] where the supertrace of nontrivial invariant functionals is demanded to be divergent.

\section{HS equations in $\boldsymbol{A d S}_{4}$}

\subsection{Nonlinear system and its cohomological interpretation}

HS theory in $A d S_{4}$ was formulated in [3] in terms of the zero-form $B(Z ; Y ; \mathcal{K} \mid x)$, space-time connection one-form $W(Z ; Y ; \mathcal{K} \mid x)$ and connection one-form $S(Z ; Y ; \mathcal{K} \mid x)$ in the $Z$-space. $W(Z ; Y ; \mathcal{K} \mid x)$ and $S(Z ; Y ; \mathcal{K} \mid x)$ can be combined into the total connection one-form

$$
\mathcal{W}=\mathrm{d}_{x}+\theta^{\underline{n}} W_{\underline{n}}(Z ; Y ; \mathcal{K} \mid x)+\theta^{A} S_{A}(Z ; Y ; \mathcal{K} \mid x), \quad \mathrm{d}_{x}=\theta^{\underline{\underline{n}}} \frac{\partial}{\partial x^{\underline{n}}},
$$

where all differentials $d Z^{A} \equiv \theta^{A}$ and $d x^{\underline{n}} \equiv \theta^{\underline{n}}$ are anticommuting. In this section, $A=$ $1, \ldots, 4$ and $\underline{n}=0, \ldots 3$ are indices of $4 d$ Majorana spinors and vectors, respectively. $\mathcal{K}=(k, \bar{k})$ denotes a pair of Klein operators that reflect chiral spinor indices of every $U^{A}=\left(u^{\alpha}, \bar{u}^{\dot{\alpha}}\right)$ with $U^{A}=\left(Y^{A}, Z^{A}, \theta^{A}\right), u^{\alpha}=\left(y^{\alpha}, z^{\alpha}, \theta^{\alpha}\right), \bar{u}^{\dot{\alpha}}=\left(\bar{y}^{\dot{\alpha}}, \bar{z}^{\dot{\alpha}}, \bar{\theta}^{\dot{\alpha}}\right)$,

$$
\begin{gathered}
k * u^{\alpha}=-u^{\alpha} * k, \quad k * \bar{u}^{\dot{\alpha}}=\bar{u}^{\dot{\alpha}} * k, \quad \bar{k} * u^{\alpha}=u^{\alpha} * \bar{k}, \quad \bar{k} * \bar{u}^{\dot{\alpha}}=-\bar{u}^{\dot{\alpha}} * \bar{k}, \\
k * k=\bar{k} * \bar{k}=1, \quad k * \bar{k}=\bar{k} * k .
\end{gathered}
$$

Note that relations (3.2) provide the definition of the star product with $k$ and $\bar{k}$. 
The simplest version of the nonlinear HS equations of [3] is

$$
\begin{aligned}
\mathcal{W} * \mathcal{W}=\mathcal{F}(\mathcal{B}), \quad \mathcal{F}(\mathcal{B}) & =-i\left(\theta_{A} \theta^{A}+\eta \delta^{2}\left(\theta_{z}\right) \mathcal{B} * k * v+\bar{\eta} \delta^{2}\left(\bar{\theta}_{\bar{z}}\right) \overline{\mathcal{B}} * \bar{k} * \bar{v}\right) \\
\mathcal{W} * \mathcal{B} & =\mathcal{B} * \mathcal{W}
\end{aligned}
$$

where

$$
\delta^{2}\left(\theta_{z}\right)=\frac{1}{2} \theta_{\alpha} \theta^{\alpha}, \quad \delta^{2}\left(\bar{\theta}_{\bar{z}}\right)=\frac{1}{2} \bar{\theta}_{\dot{\alpha}} \bar{\theta}^{\dot{\alpha}},
$$

$\eta=\exp [i \varphi], \varphi \in[0, \pi$ ) (the absolute value of $\eta$ as well as a factor of -1 can be absorbed into a redefinition of $\mathcal{B}$ ) leads to a class of pairwise nonequivalent nonlinear HS theories. The left and right inner Klein operators

$$
v=\exp i z_{\alpha} y^{\alpha}, \quad \bar{v}=\exp i \bar{z}_{\dot{\alpha}} \bar{y}^{\dot{\alpha}}
$$

commute with $\theta^{A}$ and obey

$$
\begin{array}{rlrl}
v * f(z, \bar{z} ; y, \bar{y}) & =f(-z, \bar{z} ;-y, \bar{y}) * v, & \bar{v} * f(z, \bar{z} ; y, \bar{y}) & =f(z,-\bar{z} ; y,-\bar{y}) * \bar{v} \\
v * v & =\bar{v} * \bar{v}=1 & v * \bar{v} & =\bar{v} * v .
\end{array}
$$

Equations (3.3), (3.4) can be extended to $\mathcal{W}(\theta ; Z ; Y ; \mathcal{K} \mid x)$ and $\mathcal{B}(\theta ; Z ; Y ; \mathcal{K} \mid x)$ being differential forms of arbitrary odd and even total degrees, respectively (both in $\theta_{Z}$ and in $\theta_{x}$ ). (Such an extension was considered, e.g., in $[8,9]$.) In this case equations (3.3), (3.4) are invariant under the following gauge transformations

$$
\delta \mathcal{W}=[\mathcal{W}, \varepsilon]_{*}+\xi^{N} \frac{\partial \mathcal{F}(\mathcal{B})}{\partial \mathcal{B}^{N}}, \quad \delta \mathcal{B}=\{\mathcal{W}, \xi\}_{*}+[\mathcal{B}, \varepsilon]_{*},
$$

where $\varepsilon(\theta ; Z ; Y ; \mathcal{K} \mid x)$ and $\xi(\theta ; Z ; Y ; \mathcal{K} \mid x)$, which are even and odd functions of $\theta$, respectively, are gauge parameters associated with $\mathcal{W}(\theta ; Z ; Y ; \mathcal{K} \mid x)$ and $\mathcal{B}(\theta ; Z ; Y ; \mathcal{K} \mid x) . N$ is the infinite multiindex running over all components of $\mathcal{B}$.

To clarify the origin of the Klein operators in the HS equations we rewrite eq. (3.3) in the form

$$
\mathcal{W} * \mathcal{W}=-i\left(\theta_{A} \theta^{A}+\delta^{2}\left(\theta_{z}\right) \delta^{2}(z) * \phi+\delta^{2}\left(\bar{\theta}_{\bar{z}}\right) \delta^{2}(\bar{z}) * \bar{\phi}\right)
$$

where $\phi$ and $\bar{\phi}$ commute with $\mathcal{W}$ up to $\theta_{z^{-}}$and $\bar{\theta}_{\bar{z}}$-dependent terms, respectively, that do not affect the compatibility conditions of (3.10) since $\theta_{z}^{3}=\bar{\theta}_{\bar{z}}^{3}=0$. The r.h.s. of (3.10) admits simple cohomological interpretation within the perturbative analysis of the HS equations.

Indeed, consider the standard vacuum solution with $\mathcal{B}=0$ and

$$
\mathcal{W}_{0}=\mathrm{d}_{x}+Q+W_{0}(Y \mid x),
$$

where

$$
Q:=\theta^{A} Z_{A}
$$

and the space-time one-form $W_{0}(Y \mid x)$ (the differentials $\theta_{x}$ are implicit) is some solution to the flatness equation

$$
\mathrm{d}_{x} W_{0}(Y \mid x)+W_{0}(Y \mid x) * W_{0}(Y \mid x)=0 .
$$


By eq. (2.3), the star-commutator with $Q$ is proportional to the de Rham derivative in $Z^{A}$

$$
Q * f(Z ; Y)-(-1)^{\operatorname{deg}_{f}} f(Z ; Y) * Q=-2 i \mathrm{~d}_{Z} f(Z ; Y), \quad \mathrm{d}_{Z}=\theta^{A} \frac{\partial}{\partial Z^{A}},
$$

where $\operatorname{deg}_{f}$ is the form degree of $f$. We observe that $\delta^{2}\left(\theta_{z}\right) \delta^{2}(z)$ in (3.10) describes the de Rham cohomology of $\mathrm{d}_{z} \cdot{ }^{2}$ Indeed, $\delta^{2}\left(\theta_{z}\right) \delta^{2}(z)$ is $\mathrm{d}_{Z^{-}}$closed since $\theta_{z}^{3}=0$ and is not $\mathrm{d}_{Z^{-}}$ exact since the $\delta^{2}(z)$ cannot be integrated in well-behaving functions. This means that the interaction terms on the r.h.s. of eq. (3.3) form a consistent but nontrivial source. Moreover, for the Weyl-Moyal star product, equation (3.3) admits no meaningful solution at all. Star product (2.1), that mixes $Z$ and $Y$ variables in a nontrivial way, makes system (3.3) solvable. The conjecture of section 6 suggests however that the interaction terms still cannot be removed by a local field redefinition. Note that relevance of a cohomological interpretation of the HS equations was pointed out long ago in [10].

\subsection{Perturbative analysis}

Let

$$
\mathcal{W}=\mathcal{W}_{0}+\mathcal{W}_{1}+\ldots, \quad \mathcal{B}=\mathcal{B}_{1}+\ldots,
$$

where $\mathcal{W}_{1}$ and $\mathcal{B}_{1}$ are first-order fluctuations. Linearized equations (3.3), (3.4) are

$$
\begin{aligned}
\mathrm{d} \mathcal{W}_{1}+W_{0} * \mathcal{W}_{1}+\mathcal{W}_{1} * W_{0} & =-i\left(\eta \delta^{2}\left(\theta_{z}\right) \mathcal{B}_{1} * k * v+\bar{\eta} \delta^{2}\left(\bar{\theta}_{\bar{z}}\right) \mathcal{B}_{1} * \bar{k} * \bar{v}\right), \\
\mathrm{d} \mathcal{B}_{1}+W_{0} * \mathcal{B}_{1}-\mathcal{B}_{1} * W_{0} & =0, \quad \mathrm{~d}=\mathrm{d}_{Z}+\mathrm{d}_{x} .
\end{aligned}
$$

The one-form sector of (3.17) yields

$$
\mathcal{B}_{1}^{0}(Z ; Y ; \mathcal{K} \mid x)=C^{0}(Y ; \mathcal{K} \mid x)
$$

and

$$
\mathrm{d} C^{0}(Y ; \mathcal{K} \mid x)+W_{0}(Y \mid x) * C^{0}(Y ; \mathcal{K} \mid x)-C^{0}(Y ; \mathcal{K} \mid x) * W_{0}(Y \mid x)=0,
$$

where $\mathcal{B}_{1}^{0}(Z ; Y ; \mathcal{K} \mid x)$ and $C^{0}(Y ; \mathcal{K} \mid x)$ are zero-forms.

The main tool for the perturbative analysis is provided by the standard homotopy formula

$$
\mathrm{d}_{Z} g\left(\theta_{Z} ; Z ; Y\right)=f\left(\theta_{Z} ; Z ; Y\right) \quad \Longrightarrow \quad g\left(\theta_{Z} ; Z ; Y\right)=\partial_{Z}^{*} f+\mathrm{d}_{Z} \varepsilon+g(0 ; 0 ; Y)
$$

where

$$
\partial_{Z}^{*} f:=\mathrm{d}_{Z}^{*} H(f), \quad H(f):=\int_{0}^{1} d t t^{-1} f\left(t \theta_{Z} ; t Z ; Y\right), \quad \mathrm{d}_{Z}^{*}=Z^{A} \frac{\partial}{\partial \theta^{A}} .
$$

The term $\mathrm{d}_{Z} \varepsilon$ in eq. (3.20) describes the freedom in exact forms while $g(0 ; 0 ; Y)$ represents the de Rham cohomology. Eq. (3.20) is valid provided that the homotopy integral over $t$

\footnotetext{
${ }^{2}$ This is a particular case of the de Rham cohomology associated with any submanifold $M^{\prime} \subset M$ described by the equation $F_{M^{\prime}}(X)=0$ where $X$ are local coordinates of $M$. Namely, $V_{M^{\prime}} \delta\left(F_{M^{\prime}}(X)\right)$, where $V_{M^{\prime}}$ is the volume form on $T^{*}(M) / T^{*}\left(M^{\prime}\right)$, represents the de Rham cohomology of $M$.
} 
converges, which, in accordance with the Poincaré lemma, is true if $g(0 ; 0 ; Y)=0$. Note that $\mathrm{d}_{Z}^{*} \mathrm{~d}_{Z}^{*}=0$ implies

$$
\partial_{Z}^{*} \partial_{Z}^{*}=0
$$

The two-form sector of (3.16) yields for the one-form $\mathcal{W}_{1}^{1}=\mathcal{W}_{1}^{1,0}+\mathcal{W}_{1}^{0,1}$,

$$
\begin{aligned}
& \mathcal{W}_{1}^{1,0}=\frac{1}{2} \eta \int_{0}^{1} d \tau \tau e^{i \tau z_{\alpha} y^{\alpha}} z_{\alpha} \theta^{\alpha} C(-\tau z, \bar{y} ; \mathcal{K}) k+\frac{1}{2} \bar{\eta} \int_{0}^{1} d \bar{\tau} \bar{\tau} \exp ^{i \bar{\tau} \bar{z}_{\dot{\alpha}} \bar{y}^{\dot{\alpha}}} \bar{z}_{\dot{\alpha}} \bar{\theta}^{\dot{\alpha}} C(y,-\bar{\tau} \bar{z} ; \mathcal{K}) \bar{k} \\
& \mathcal{W}_{1}^{0,1}=\omega^{1}-\frac{i}{2} \partial_{Z}^{*}\left\{W_{0}, \mathcal{W}_{1}^{1,0}\right\}
\end{aligned}
$$

where the one-form $\omega^{1}\left(\theta_{x} ; Y ; \mathcal{K} \mid x\right)$ describes physical HS gauge fields valued in the cohomology of $\mathrm{d}_{Z}$ (the term with $\mathrm{d}_{x}$ in (3.16) does not contribute to the second term in (3.24) due to (3.22)). Plugging (3.24) into the $\theta_{x}^{2}$ sector of (3.16) yields the so-called First On-Shell Theorem which imposes dynamical equations on the spin $s>1$ frame-like fields contained in $\omega^{1}\left(\theta_{x} ; Y ; \mathcal{K} \mid x\right)$ (for more detail see $[11]$ and references therein).

The aim of this section is to recall two general features.

The first is that dynamically nontrivial (physical) components of the HS fields like $C^{0}(Y ; \mathcal{K} \mid x)$ and $\omega^{1}\left(\theta_{x} ; Y ; \mathcal{K} \mid x\right)$ are in the $\mathrm{d}_{Z}$ cohomology. Other components of the HS fields are expressed via the physical components by the HS equations. Indeed, since d contains $\mathrm{d}_{\mathrm{Z}}$ (3.14), equations (3.16) and (3.17) express all components of $\mathcal{W}_{1}$ and $\mathcal{B}_{1}$ that are not $\mathrm{d}_{\mathrm{Z}}$ closed via other fields. $\mathrm{d}_{\mathrm{Z}}$-exact fields are pure gauge with respect to the gauge transformations (3.9). Hence, the remaining physical fields are in the $\mathrm{d}_{\mathrm{Z}}$-cohomology. By Poincaré Lemma, physical fields are independent of both $Z^{A}$ and $\theta^{A}$, i.e.,

$$
C(Y ; \mathcal{K} \mid x):=\left.\mathcal{B}_{1}(Z ; Y ; \mathcal{K} \mid x)\right|_{\theta_{Z}=Z=0}, \quad \omega\left(\theta_{x} ; Y ; \mathcal{K} \mid x\right):=\left.\mathcal{W}_{1}(\theta ; Z ; Y ; \mathcal{K} \mid x)\right|_{\theta_{Z}=Z=0}
$$

(Note that this consideration ignores the issue controlled by the so-called $\sigma_{-}$-cohomology that the HS equations impose further constraints expressing some of the physical fields via space-time derivatives of the other.)

The second is that the perturbative solution of the HS equations leads to homotopy integrals over $\tau$ in formulae like (3.23). Higher perturbations lead to multiple homotopy integrals which are in the core of the analysis of star-product functional classes in the sequel.

\section{Functional spaces}

\subsection{Algebra $V_{0,0}$}

Functions of the variables $Y$ and $Z$, emerging in the perturbative analysis of HS equations, are nonpolynomial. Indeed, application of (3.20) to terms containing the Klein operators (3.6) gives rise to functions of the form

$$
f(Z ; Y)=\int_{0}^{1} d \tau \varphi(Z ; Y ; \tau) \exp i \tau Z_{A} Y^{A}
$$


where indices $A, B, \ldots=1,2, \ldots, M$ can take any even number of values (e.g., $Z_{A}, Y_{A}$ can denote each of the pairs of spinors $z_{\alpha}, y_{\alpha}$ or $\left.\bar{z}_{\dot{\alpha}}, \bar{y}_{\dot{\alpha}}\right)$ and

$$
\varphi(Z ; Y ; \tau)=\sum_{n, m=0}^{\infty} \varphi_{A_{1}, \ldots, A_{n}, B_{1}, \ldots, B_{m}}(\tau) Z^{A_{1}} \ldots Z^{A_{n}} Y^{B_{1}} \ldots Y^{B_{m}}
$$

is a polynomial or power series in $Z, Y$ with the coefficients $\varphi_{A_{1}, \ldots, A_{n}, B_{1}, \ldots, B_{m}}(\tau)$ integrable in $\tau$. Distributions in $\tau$ are also allowed.

As observed originally in [12], elements (4.1) form a closed algebra under the HS star product (2.1), i.e., their star products are free of divergencies and belong to the class (4.1). This is specific for the HS star product (2.1) and may not be true for other star products associated with different ordering prescriptions. Indeed, a possible divergency of the star product of Gaussian exponentials is due to a potential degeneracy of the Gaussian bilinear form in the integration variables $U$ and $V$ in (2.1). However, because of the form of star product (2.1), the $\tau$-dependent exponential in (4.1) does not contribute to the quadratic part in $U$ and $V$ since $U_{A} U^{A}=V_{A} V^{A}=0$. For other star products, which typically involve $4 M$ integration variables in the analogue of (2.1) (cf. formula (B.3) for the Weyl star product), this mechanism does not work and divergencies can appear.

An elementary computation yields

$$
\left(f_{1} * f_{2}\right)(Z ; Y)=\int d \tau_{1,2} \varphi_{1,2}\left(Z ; Y ; \tau_{1,2}\right) \exp i \tau_{1,2} Z_{A} Y^{A},
$$

where

$$
\begin{aligned}
\varphi_{1,2}\left(Z ; Y ; \tau_{1,2}\right)= & \frac{1}{(2 \pi)^{M}} \int d \tau_{1} d \tau_{2} d S d T \delta\left(\tau_{1,2}-\tau_{1} \diamond \tau_{2}\right) \exp i S_{A} T^{A} \\
& \varphi_{1}\left(\left(1-\tau_{2}\right) Z-\tau_{2} Y+S ;\left(1-\tau_{2}\right) Y-\tau_{2} Z+S ; \tau_{1}\right) \\
& \varphi_{2}\left(\left(1-\tau_{1}\right) Z+\tau_{1} Y-T ; \tau_{1} Z+\left(1-\tau_{1}\right) Y+T ; \tau_{2}\right),
\end{aligned}
$$

and

$$
a \diamond b=a+b-2 a b=a(1-b)+b(1-a)
$$

can be interpreted as a product in $\mathbb{R}$ or $\mathbb{C}$. It is obviously commutative

$$
a \diamond b=b \diamond a
$$

and associative, being inherited from the associative star product. Setting

$$
a=\frac{1}{2}(1-\alpha), \quad b=\frac{1}{2}(1-\beta)
$$

(4.5) yields the usual product $\alpha \beta$ with $a=0, \frac{1}{2}$ and 1 mapped, respectively, to $\alpha=1,0$, and -1 . This complies with the facts that, with respect to the star product, 1 is the unit element, $\exp i Z_{A} Y^{A}$ is the involutive Klein operator (2.6) and

$$
F=\exp \frac{i}{2} Z_{A} Y^{A}
$$


is the Fock vacuum obeying

$$
\left(Y_{A}+Z_{A}\right) * F=0, \quad F *\left(Y_{A}-Z_{A}\right)=0, \quad F * \exp \left(i a Z_{A} Y^{A}\right)=\exp \left(i a Z_{A} Y^{A}\right) * F=F
$$

Since the segment $[-1,1]$ is invariant under multiplication, it follows that

$$
a, b \in[0,1] \quad \Longrightarrow \quad a \diamond b \in[0,1]
$$

as is also obvious from

$$
1-a \diamond b=(1-a)(1-b)+a b .
$$

For $\varphi_{1}(Z ; Y ; \tau)$ and $\varphi_{2}(Z ; Y ; \tau)$ polynomial in $Z$ and $Y$, that are integrable in $\tau, \varphi_{1,2}$ is also a polynomial integrable in $\tau_{1,2}$. Hence functions $(4.1)$ form an algebra called $\mathcal{H}^{\text {tot }}$.

Consider the subspace $V_{0,0} \subset \mathcal{H}^{\text {tot }}$ of functions of the form

$$
f(Z ; Y)=\int_{0}^{1} d \tau \phi(\tau Z ;(1-\tau) Y ; \tau) \exp i \tau Z_{A} Y^{A}
$$

with $\phi(W ; U ; \tau)$ regular in $W$ and $U$ and integrable in $\tau$. Being accompanied by the factor of $\tau$ and $1-\tau$, the dependence on $Z$ and $Y$ on the r.h.s. of (4.12) trivializes at $\tau \rightarrow 0$ and $\tau \rightarrow 1$, respectively. Such a behavior turns out to be most appropriate for the perturbative analysis of the HS theory.

The functions $\phi(W, U, \tau)$ localized at $\tau=0$ or $\tau=1$ are of special interest. Functions (4.12) with $\phi(W ; U ; \tau)$ proportional to $\delta(\tau)$ yield $Z$-independent elements

$$
f(Z ; Y)=g(Y)
$$

while those proportional to $\delta(1-\tau)$ have the form

$$
f(Z ; Y)=h(Z) \exp i Z_{A} Y^{A} .
$$

The following remarkable fact is true:

Theorem 1: $V_{0,0}$ forms an associative algebra with respect to the star product (2.1). Elements with $\phi(W ; U ; \tau)$ polynomial in $W, U$ form its subalgebra.

For the proof we observe that (4.4) yields

$$
\begin{aligned}
\varphi_{1,2}\left(W ; U ; \tau_{1,2}\right)= & \frac{1}{(2 \pi)^{M}} \int d S d T \exp i S_{A} T^{A} \int_{0}^{1} d \tau_{1} d \tau_{2} \delta\left(\tau_{1,2}-\tau_{1} \diamond \tau_{2}\right) \\
& \phi_{1}\left(\tau_{1}\left[\left(1-\tau_{2}\right) W-\tau_{2} U+S\right] ;\left(1-\tau_{1}\right)\left[\left(1-\tau_{2}\right) U-\tau_{2} W+S\right] ; \tau_{1}\right) \\
& \phi_{2}\left(\tau_{2}\left[\left(1-\tau_{1}\right) W+\tau_{1} U-T\right] ;\left(1-\tau_{2}\right)\left[\tau_{1} W+\left(1-\tau_{1}\right) U+T\right] ; \tau_{2}\right) .
\end{aligned}
$$

Elementary inequalities following from eqs. (4.5) and (4.11)

$$
\begin{array}{rlrl}
\tau_{1} \diamond \tau_{2} & \geq\left(1-\tau_{1}\right) \tau_{2} \geq 0 & \tau_{1} \diamond \tau_{2} & \geq\left(1-\tau_{2}\right) \tau_{1} \geq 0, \\
1-\tau_{1} \diamond \tau_{2} & \geq\left(1-\tau_{1}\right)\left(1-\tau_{2}\right) \geq 0 & 1-\tau_{1} \diamond \tau_{2} \geq \tau_{1} \tau_{2} \geq 0
\end{array}
$$


imply that

$$
\begin{aligned}
\left(1-\tau_{1}\right) \tau_{2} & =\alpha\left(\tau_{1}, \tau_{2}\right) \tau_{1} \diamond \tau_{2} & \left(1-\tau_{2}\right) \tau_{1} & =\beta\left(\tau_{1}, \tau_{2}\right) \tau_{1} \diamond \tau_{2}, \\
\left(1-\tau_{1}\right)\left(1-\tau_{2}\right) & =\gamma\left(\tau_{1}, \tau_{2}\right)\left(1-\tau_{1} \diamond \tau_{2}\right) & \tau_{1} \tau_{2} & =\rho\left(\tau_{1}, \tau_{2}\right)\left(1-\tau_{1} \diamond \tau_{2}\right)
\end{aligned}
$$

with

$$
\alpha\left(\tau_{1}, \tau_{2}\right), \beta\left(\tau_{1}, \tau_{2}\right), \gamma\left(\tau_{1}, \tau_{2}\right), \rho\left(\tau_{1}, \tau_{2}\right) \in[0,1] .
$$

This implies that, for any $f_{1}$ and $f_{2}$ of the form (4.12), $f_{1} * f_{2}$ also has the form (4.12) with

$$
\begin{aligned}
\phi_{1,2}(W ; U ; \tau)= & \frac{1}{(2 \pi)^{M}} \int d S d T \exp i S_{A} T^{A} \int_{0}^{1} d \tau_{1} d \tau_{2} \delta\left(\tau-\tau_{1} \diamond \tau_{2}\right) \\
& \phi_{1}\left(\beta W-\rho U+\tau_{1} S ; \gamma U-\alpha W+\left(1-\tau_{1}\right) S ; \tau_{1}\right) \\
& \phi_{2}\left(\alpha W+\rho U-\tau_{2} T ; \beta W+\gamma U+\left(1-\tau_{2}\right) T ; \tau_{2}\right)
\end{aligned}
$$

provided that

$$
\int d S d T \exp i S_{A} T^{A} \phi_{1}\left(W+a S ; U+(1-a) S ; \tau_{1}\right) \phi_{2}\left(V-b T ; Y+(1-b) T ; \tau_{2}\right), \quad a, b \in[0,1]
$$

is well defined (converges). Due to (4.20), $\phi_{1,2}(W, U, \tau)$ is integrable in $\tau$. Hence, $f_{1} * f_{2} \in$ $V_{0,0}$. Clearly, $\phi_{1,2}(W, U, \tau)$ is polynomial in $W$ and $U$ if $\phi_{1}(W ; U ; \tau)$ and $\phi_{2}(W ; U ; \tau)$ were

The property inherited from the Klein operator is that not every element of $V_{0,0}$ admits supertrace. Indeed, by virtue of (2.4),

$$
\operatorname{str}(f)=\frac{1}{(2 \pi)^{M}} \int d^{M} Z d^{M} Y \int_{0}^{1} d \tau \phi(\tau Z ;(1-\tau) Y ; \tau) \exp -i(1-\tau) Z_{A} Y^{A} .
$$

Changing the integration variables $Y^{A}=(1-\tau)^{-1} U^{A}, Z^{A}=V^{A}$ yields

$$
\operatorname{str}(f)=\frac{1}{(2 \pi)^{M}} \int d^{M} U d^{M} V \int_{0}^{1} d \tau(1-\tau)^{-M} \phi(\tau V ; U ; \tau) \exp -i\left(V_{A} U^{A}\right) .
$$

Since $\phi(W ; U ; \tau)$ is regular in its arguments, $\operatorname{str}(f)$ is well defined provided that the integral over $\tau$ converges at $\tau=1$, i.e., $\phi(V ; U ; \tau)$ appropriately tends to zero at $\tau \rightarrow 1$. In section 5 it is shown that, generally, only the logarithmic divergency matters, and the algebra $\mathcal{H}_{0}^{\text {loc }}$ free of trace divergencies is introduced.

Let us stress that, beyond the algebra $V_{0,0}$, the degree of divergency of the supertrace may depend on the degree of $Y$ (i.e., spin) while in $V_{0,0}$ this does not happen just because the $Y$-dependence in (4.12) has been rescaled by $1-\tau$.

\subsection{Spaces $V_{k, l}$}

It is useful to consider the space $V_{k, l}$ of such star-product elements $(4.12)$ that $\phi(W ; U ; \tau)$ scales as $\tau^{k}$ at $\tau \rightarrow 0$ and $(1-\tau)^{l}$ at $\tau \rightarrow 1$. More precisely, we allow (poly)logarithmic dependence on $\tau$ and $1-\tau$ at $\tau \rightarrow 0$ and $\tau \rightarrow 1$, respectively, with the convention that it does not affect the indices $k$ and $l$. We consider $V_{k, l}$ with both positive and negative 
$k$ and/or $l$ assuming however that the function $\varphi(Z ; Y ; \tau)$ in $(4.1)$ is integrable in $\tau$. For example, for the spaces $V_{-1,0}$ and $V_{0,-1}$ this is the case if $\phi(0 ; U ; \tau)=0$ and $\phi(W ; 0 ; \tau)=0$, respectively.

Thus, $V_{k, l}$ contains such $f(4.12)$ that $\tau^{-k}(1-\tau)^{-l} \phi(W ; U ; \tau)$ has non-negative scalings at $\tau \rightarrow 0,1$. The spaces $V_{k, l}$ with non-integer indices $k$ and $l$ are also allowed. With this definition

$$
V_{k+\epsilon, l} \subset V_{k, l}, \quad V_{k, l+\epsilon} \subset V_{k, l} \quad \forall \epsilon>0 .
$$

Via the decomposition $1=\tau+(1-\tau)$ any $f \in V_{k, l}$ can be represented in the form

$$
f \in V_{k, l}: \quad f=g+h, \quad g \in V_{k+1, l}, \quad h \in V_{k, l+1} .
$$

Repeated application of this formula along with (4.25) gives

\section{Lemma 1:}

$$
V_{k, l}=V_{k, \infty} \cup V_{\infty, l},
$$

where

$$
V_{k, \infty} \subset V_{k, l} \quad \forall l, \quad V_{\infty, l} \subset V_{k, l} \quad \forall k,
$$

i.e., elements of $V_{k, \infty}\left(V_{\infty, l}\right)$ have $\phi(W ; U ; \tau)$ that tend to zero at $\tau \rightarrow 1(\tau \rightarrow 0)$ faster than any power of $1-\tau(\tau)$.

Alternatively, Lemma 1 follows from the decomposition

$$
\begin{aligned}
\int_{0}^{1} d \tau \phi(\tau) & =\int_{-\infty}^{\infty} d \tau \vartheta(\tau) \vartheta(1-\tau) \phi(\tau), \\
\vartheta(\tau) \vartheta(1-\tau) \phi(W ; U ; \tau) & =\vartheta(\tau) \vartheta(a-\tau) \phi(W ; U ; \tau)+\vartheta(\tau-a) \vartheta(1-\tau) \phi(W ; U ; \tau) \quad \forall a \in(0,1),
\end{aligned}
$$

where $\vartheta(\tau)$ is the step function. Indeed, here the first and second terms are identically zero in some neighborhood of $\tau=1$ and $\tau=0$, respectively.

For distributions we assign that

$$
\begin{array}{lll}
\phi(W ; U ; \tau) & \sim \delta(\tau): & f(Z ; Y) \in V_{-1, \infty} \\
\phi(W ; U ; \tau) \sim \delta(1-\tau): & & f(Z ; Y) \in V_{\infty,-1} .
\end{array}
$$

The derivatives $\delta^{k}(\tau)$ and $\delta^{k}(1-\tau)$ of $\delta(\tau)$ and $\delta(1-\tau)$ are assigned to $V_{-1-k, \infty}$ and $V_{\infty,-1-k,}$, respectively.

The spaces $V_{k, l}$ have the fundamental composition property expressed by

\section{Theorem 2:}

$$
V_{k_{1}, l_{1}} * V_{k_{2}, l_{2}} \subset V_{\min \left(k_{1}, l_{2}\right)+\min \left(k_{2}, l_{1}\right)+1, \min \left(k_{1}, k_{2}\right)+\min \left(l_{1}, l_{2}\right)+1} .
$$


This follows from formula (4.15) and inequalities (4.16), (4.17) along with the simple fact that the integral

$$
\int_{0}^{1} d \tau_{1} \int_{0}^{1} d \tau_{2} \delta\left(\tau-\tau_{1} \diamond \tau_{2}\right)=-\log \left((1-2 \tau)^{2}\right)
$$

behaves as $\tau$ at $\tau \rightarrow 0$ and $1-\tau$ at $\tau \rightarrow 1$

Formula (4.33) has useful consequences. In particular

$$
V_{k, l} * V_{k, l} \subset V_{2 \min (k, l)+1, k+l+1} .
$$

For $V_{k, l}$ with $k \geq-1, l \geq-1$ from here it follows using (4.25) that

$$
V_{k, l} * V_{k, l} \subset V_{\min (k, l), \max (k, l)}, \quad k \geq-1, l \geq-1 .
$$

Hence, $V_{k, l}$ with $l \geq k \geq-1$ forms an algebra

$$
V_{k, l} * V_{k, l} \subset V_{k, l}, \quad l \geq k \geq-1 .
$$

The fact proven in section 4.1 that $V_{0,0}$ is an algebra is a particular case of (4.36).

From (4.25), (4.33) and (4.35) it follows that

$$
V_{l, k} * V_{k, l} \subset V_{l, k}, \quad V_{k, l} * V_{l, k} \subset V_{l, k}, \quad V_{l, k} * V_{l, k} \subset V_{k, l}, \quad l \geq k \geq-1,
$$

implying along with (4.36) that

$$
W_{l, k}=W_{k, l}:=V_{k, l} \cup V_{l, k}, \quad k \geq-1, l \geq-1
$$

is an algebra. Interesting algebras of this type are $W_{-1,0}$ and $W_{-1, \infty}$.

For $f(4.12)$, formula (4.15) with $f_{2}=\Upsilon$ yields

$$
f * \Upsilon=\int_{0}^{1} d \tau \phi(-(1-\tau) Y ;-\tau Z ; 1-\tau) \exp i \tau Z_{A} Y^{A} .
$$

Along with (2.8) this has an important consequence

$$
\Upsilon * V_{k, l}=V_{k, l} * \Upsilon=V_{l, k} .
$$

Together with (4.33) this gives

\section{Lemma 2:}

$$
\begin{aligned}
& V_{-1, \infty} * V_{k, l}=V_{k, l} * V_{-1, \infty}=V_{k, l}, \\
& V_{\infty,-1} * V_{k, l}=V_{k, l} * V_{\infty,-1}=V_{l, k} .
\end{aligned}
$$

From (4.41) it follows in particular that the star product of a function that depends only on $Y$ with an element of $V_{k, l}$ belongs to $V_{k, l}$. 


\subsection{The derivative and homotopy}

Now we extend consideration to differential forms in the $Z$ space. Let $f\left(\theta_{Z} ; Z ; Y\right) \in V_{k, l, p}$ if $f\left(\theta_{Z} ; Z ; Y\right)$ is a $p$-form with coefficients in $V_{k, l}$.

An important property of $Q(3.12)$ is

Lemma 3: $Q \in V_{-2, \infty, 1}$.

This is because

$$
Q=\int_{0}^{1} d \tau \delta(\tau) \tau^{-1}\left(\tau \theta^{A} Z_{A}\right)
$$

where both $\delta(\tau)$ and $\tau^{-1}$ bring negative contribution to the first index of $V_{-2, l, 1}$.

Since the graded star-commutator $[Q, \ldots]_{*}$ is equivalent to $\mathrm{d}_{Z}=\theta^{A} \frac{\partial}{\partial Z^{A}}$, we arrive at

Lemma 4: $\left[Q, V_{k, l, p}\right]_{*} \subset V_{k+1, l-1, p+1}$.

Here $k$ increases because the $Z$-differentiation of $f(Z ; Y)(4.12)$ brings one power of $\tau$ while $l$ decreases because the $Z$-differentiation of the exponential in (4.12) brings one power of $Y$ requiring a factor of $1-\tau$

Suppose that $f \in V_{k, l, p}$ is $Q$-closed. By homotopy formula (3.21), a solution to equation $(3.20) \mathrm{d}_{Z} g=f$ for $f(4.12)$ is

$$
\partial_{Z}^{*} f\left(\theta_{Z} ; Z ; Y\right)=Z^{A} \frac{\partial}{\partial \theta^{A}} \int_{0}^{1} \frac{d s}{s} \int_{0}^{1} d \tau \phi\left(s \theta_{Z} ; s \tau Z ;(1-\tau) Y ; \tau\right) \exp i s \tau Z_{A} Y^{A} .
$$

An elementary analysis sketched in appendix A proves

Lemma 5:

$$
\partial_{Z}^{*} V_{k, l, p} \subset V_{\min (p-1, k)-1, l+1, p-1}
$$

\subsection{Inner and boundary spaces}

To distinguish between $\phi(W ; U ; \tau)$ localized at the boundary of the segment $\tau \in[0,1]$ and those smooth at $\tau=0$ or $\tau=1$, the functions $\phi(W ; U ; \tau)$ should be further specified. Factors of $W_{A} U^{A}$ in $\phi(W ; U ; \tau)$ can be removed by the partial integration over $\tau$ in (4.12) implying that

$$
\exp \left(i \tau Z_{A} Y^{A}\right)\left[i \vartheta(\tau) \vartheta(1-\tau) Z_{A} Y^{A} \chi(\tau Z ;(1-\tau) Y ; \tau)+\frac{\partial}{\partial \tau}(\vartheta(\tau) \vartheta(1-\tau) \chi(\tau Z ;(1-\tau) Y ; \tau))\right] \sim 0
$$

As a result, the representation (4.12) can be achieved with such $\phi(W ; U ; \tau)$ that its inner part $\phi^{\text {in }}(W ; U ; \tau)$, containing an additional factor of $\tau(1-\tau)$ to cancel the denominator in

$$
Z_{A} Y^{A}=\tau^{-1}(1-\tau)^{-1} W_{A} U^{A}, \quad W_{A}=\tau Z_{A}, \quad U_{A}=(1-\tau) Y_{A},
$$


obeys the condition

$$
\frac{\partial^{2}}{\partial W_{A} \partial U^{A}} \phi^{\text {in }}(W ; U ; \tau)=0 .
$$

The spaces of $f \in V_{k, l}$ (4.12) with smooth functions $\phi(W ; U ; \tau)$ obeying (4.48) and those localized at $\tau=0$ or 1 will be called inner space $\widetilde{V}_{k, l}$ and boundary space $\bar{V}_{k, l}$, respectively. They can play a róle in the analysis of HS dynamics as discussed in section 7 . Taking into account the dependence on $\tau$, condition (4.48) restricts $\phi(W ; U ; \tau)$ to functions of as many variables as the original unrestricted function $f(Z ; Y)$, i.e., $\widetilde{V}_{k, l}$ is as large as the space of functions of $Z, Y$. On the other hand, $\bar{V}_{k, l}$ is the space of functions that depend either only on $Y$ or only on $Z$ since the dependence on $Z$ and $Y$ in (4.12) trivializes at $\tau=0$ and $\tau=1$, respectively (cf. eqs. (4.13), (4.14)).

In particular, physical fields defined in section 3.2 as belonging to $\mathrm{d}_{Z}$-cohomology obey

Lemma 6: Physical fields belong to $\overline{\mathcal{H}}^{\text {phys }}:=\bar{V}_{-1, \infty, 0} . \overline{\mathcal{H}}^{\text {phys }}$ is an algebra.

\section{HS field algebra $\mathcal{H}$ and local algebra $\mathcal{H}^{l o c}$}

The case with a single homotopy parameter $\tau$ in (4.12) considered so far is not most general since, like in HS equations (3.3), there may be several Klein operators associated with different de Rham cohomologies in the $Z$-space. In that case we introduce several homotopy parameters $\vec{\tau}=\left(\tau_{1}, \tau_{2} \ldots\right)$ with the respective multiindices $\vec{k}=\left(k_{1}, k_{2}, \ldots\right)$, $\vec{l}=\left(l_{1}, l_{2}, \ldots\right)$ and $\vec{p}=\left(p_{1}, p_{2}, \ldots\right)$ of $V_{\vec{k}, \vec{l}, \vec{p}}$. If the homotopy parameters appear in the combination $\exp i \tau Z_{A} Y^{A}$, for the example of $4 d$ HS model we set

$$
\int_{0}^{1} d \tau \varphi(Z ; Y ; \tau) \exp i \tau Z_{A} Y^{A}=\int_{0}^{1} d \tau_{1} \int_{0}^{1} d \tau_{2} \varphi\left(Z ; Y ; \tau_{1}\right) \delta\left(\tau_{1}-\tau_{2}\right) \exp i\left[\tau_{1} z_{\alpha} y^{\alpha}+\tau_{2} \bar{z}_{\dot{\alpha}} \bar{y}^{\dot{\alpha}}\right],
$$

assigning the indices $k_{1}, k_{2}$ and $l_{1}, l_{2}$ freely at the condition that $k_{1}+k_{2}=k$ and $l_{1}+l_{2}=l$ where $k$ and $l$ control the behavior in $\tau$. With these multi-index notations our analysis applies to general HS systems with $M \rightarrow \vec{M}=\left(M_{1}, M_{2}, \ldots\right), M=M_{1}+M_{2}+\ldots$ For the sake of simplicity we will use the single-index notation in the sequel.

\subsection{HS field algebra}

The results of sections 4.2 and 4.3 allow us to identify the space of fields $\mathcal{H}$ appropriate for the perturbative analysis of the HS equations:

$$
\mathcal{H}:=\oplus_{p=0}^{M} \mathcal{H}_{p}, \quad \mathcal{H}_{p}:=V_{p-1, M-p-1, p} .
$$

Using that any $p$-form in $\theta_{Z}$ with $p>M$ is zero, from formula (4.33) at $p+q \leq M$ follows

Lemma 7: $\mathcal{H}_{p} * \mathcal{H}_{q} \subset \mathcal{H}_{p+q}$.

Lemma 7 respects the $\mathbb{Z}$-grading of the exterior algebra and implies the important 
Theorem 3: $\mathcal{H}$ is an algebra, i.e., $\mathcal{H} * \mathcal{H} \subset \mathcal{H}$.

$\mathcal{H}$ will be called $H S$ field algebra. Note that by Lemma 6 physical fields belong to $\overline{\mathcal{H}}^{\text {phys }} \subset \mathcal{H}_{0} \subset \mathcal{H}$.

Lemmas 2 and 3 imply

Lemma 8: $\left[Q, \mathcal{H}_{p}\right]_{*} \subset \mathcal{H}_{p+1}, \quad \partial_{Z}^{*} \mathcal{H}_{p} \subset \mathcal{H}_{p-1}, \quad \partial_{Z}^{*} \mathcal{H}_{0}=0$

which has a consequence

Theorem $4: \mathcal{H}$ is invariant under the action of the homotopy operator $\partial_{Z}^{*}$ and derivative $\mathrm{d}_{Z}$.

One of the important conclusions of this paper is that the operator $Q \in V_{-2, \forall l, 1}$ does not belong to $\mathcal{H}$. Theorem 4 then implies that $Q$ induces an outer derivation of $\mathcal{H}$. Correspondingly, the HS connection $\mathcal{W}$ should be written in the form

$$
\mathcal{W}=\mathrm{d}_{x}+Q+\mathcal{W}^{\prime}, \quad \mathcal{W}^{\prime} \in \mathcal{H}
$$

The central result of this section is

Theorem 5: The fields $\mathcal{W}^{\prime}$ and $\mathcal{B}$ resulting from the perturbative solution of the HS equation with the homotopy operator (3.21) belong to $\mathcal{H}$ in all orders of the perturbative expansion.

The proof follows from Theorems 3, 4 along with the fact that, since $\delta^{M}\left(\theta_{Z}\right) \Upsilon \in$ $\mathcal{H}_{M} \subset \mathcal{H}$, by Theorem 3 the term $\delta^{M}\left(\theta_{Z}\right) \mathcal{B} * k * \Upsilon$ on the r.h.s. of the HS equations belongs to $\mathcal{H}$. Also one should take into account that the physical fields belong to $\mathcal{H}$ by Lemma 6

Another important consequence of Theorems 3 and 4 is

Theorem 6: Gauge transformations (3.9) with $\varepsilon, \xi \in \mathcal{H}$ leave the HS fields in $\mathcal{H}$.

A distinguishing property of the homotopy operator $\partial_{Z}^{*}$ is that it maps the spaces $V_{k, l, p}$ in accordance with (4.45). Generally, this is not automatic because $g$ in (3.20) is reconstructed up to exact forms which can a priori belong to other spaces $V_{n, m, p}$. For instance, the homotopy operators defined with respect to $Z \pm Y$ rather than $Z$, which were used in the early works on HS interactions (see e.g. [10]), do not respect the filtration of the spaces $V_{k, l, p}$ and do not fit the above scheme. A posteriori, we realize that it is property (4.45) that to large extent determines the algebraic setup underlying the HS equations.

\subsection{Local HS algebra}

A local HS algebra $\mathcal{H}^{l o c} \subset \mathcal{H}$ is defined as follows

$$
\mathcal{H}^{l o c}=\oplus_{p=0}^{M} \mathcal{H}_{p}^{l o c}, \quad \mathcal{H}_{p}^{l o c} \subset V_{p-1, M-p-1+\epsilon, p}, \quad \forall \epsilon>0 .
$$


The difference between $\mathcal{H}^{l o c}$ and $\mathcal{H}$ is dominated by any rational behavior in $1-\tau$. From Lemmas 4,5 and Theorem 2 the analogues of Lemma 7 and Theorems 3,4 follow

Lemma 7': $\mathcal{H}_{p}^{l o c} * \mathcal{H}_{q}^{l o c} \subset \mathcal{H}_{p+q}^{l o c}$.

Theorem $3^{\prime}: \mathcal{H}^{l o c}$ is an algebra.

Theorem $4^{\prime}: \mathcal{H}^{l o c}$ is invariant under the action of the homotopy operator $\partial_{Z}^{*}$ and $\mathrm{d}_{Z}$.

From eq. (4.24) it follows that the supertrace of elements of $\mathcal{H}_{p}$ diverges as

$$
\operatorname{str}\left(\mathcal{H}_{p}\right) \sim \int_{0}^{1} d \tau(1-\tau)^{-1-p} \ldots
$$

In particular, the supertrace of an element of $\mathcal{H}_{0}$ diverges at most logarithmically. The supertraces of elements of $\mathcal{H}_{p}^{\text {loc }}$ with $p>0$ diverge analogously to $\mathcal{H}_{p}$. However elements of $\mathcal{H}_{0}^{l o c}$ have well-defined supertrace. By Lemma $7^{\prime}$ we arrive at

Theorem 7: $\mathcal{H}_{0}^{\text {loc }}$ is an algebra endowed with the well defined supertrace for elements (4.12) with

$$
\phi(W ; U ; \tau)=\tau^{-1}(1-\tau)^{M-1+\epsilon} \phi^{\prime}(W ; U ; \tau)
$$

obeying

$$
\int d^{M} U d^{M} V \phi^{\prime}(V ; U ; \tau) \exp -i\left(V_{A} U^{A}\right)<\infty .
$$

In particular, (5.7) holds for any $\phi^{\prime}(V ; U ; \tau)$ polynomial in $V$ and $U$.

The algebras $\mathcal{H}_{0}$ and $\mathcal{H}_{0}^{\text {loc }}$ play an important róle in the analysis of [7] where invariant functionals of the $3 d$ and $4 d$ HS theories are constructed as certain projections of combinations of HS fields in the $\theta_{Z}$-independent sector, that should have divergent supertrace, hence belonging to $\mathcal{H}_{0} / \mathcal{H}_{0}^{\text {loc }}$.

It is also useful to introduce the ultralocal algebra $\mathcal{H}^{\text {ult }}$

$$
\mathcal{H}^{\text {ult }}=\oplus_{p=0}^{M} \mathcal{H}_{p}^{u l t}, \quad \mathcal{H}_{p}^{u l t} \subset V_{p-1, \infty, p}
$$

This contains the boundary subalgebra of physical fields $\overline{\mathcal{H}}^{\text {phys }} \subset \mathcal{H}_{0}^{\text {ult }}$.

\section{$6 \quad$ Locality conjecture}

Results of section 5 lead to a conjecture that may help to distinguish between local and nonlocal functionals and field redefinitions in the HS theory. Namely, consider a perturbative function of the fields $\phi=\mathcal{W}^{\prime}, \mathcal{B} \in \mathcal{H}$

$$
f(\phi)=f+\sum_{g, h, \ldots}\left(g_{1} * \phi * g_{2}+h_{1} * \phi * h_{2} * \phi * h_{3}+\ldots\right),
$$


where summation is over various $f, g, h, \ldots$. We call $f(\phi)$ local if all $f, g, h, \ldots \in \mathcal{H}^{\text {loc }}$, minimally nonlocal if $f, g, h, \ldots \in \mathcal{H}$ and strongly nonlocal otherwise. Analogous terminology applies to field redefinitions $\phi \rightarrow \phi^{\prime}=f(\phi)$. Clearly, since $(\mathcal{H}) \mathcal{H}^{l o c}$ is an algebra, the composition of any two (minimally nonlocal) local transformations (6.1) is (minimally nonlocal) local. Since $\mathcal{H}^{l o c} \subset \mathcal{H}$, any local transformation is minimally nonlocal.

The conjecture is that, in the HS theory, so defined local transformations provide a proper generalization of the local transformations in Minkowski space. We will call transformations strongly local if $f, g, h, \ldots \in \mathcal{H}^{l o c}$ are supported by polynomial $\phi(W ; U ; \tau)$ in (4.12). (Recall that, by Theorem 1, such elements form an algebra.) Analogously, transformations (6.1) will be called (strongly) ultralocal for (polynomial) $f, g, h, \ldots \in \mathcal{H}^{\text {ult }}$. The most restrictive class is with polynomial $f, g, h, \ldots \in \overline{\mathcal{H}}^{\text {phys }}$. Maximally local maps of this class are the closest analogues of the local maps in Minkowski space, describing usual local field redefinitions of the physical fields like $\omega^{1}(3.24)$ and $C^{0}(3.18)$.

The rationale behind the locality conjecture is that, as discussed in section 1 , by virtue of the unfolded equations the behavior in the twistor-like variables $Z^{A}$ and $Y^{A}$ effectively encodes the space-time derivative expansion of the dynamical fields hidden in $\phi$. For $f \in V_{k, l}$ (4.12) with

$$
\phi\left(\theta_{Z} ; W ; U ; \tau\right)=\tau^{k}(1-\tau)^{l} \phi^{\prime}\left(\theta_{Z} ; W ; U ; \tau\right),
$$

where, schematically,

$$
\phi^{\prime}\left(\theta_{Z} ; W ; U ; \tau\right)=\sum_{n, m} a_{n m}\left(\theta_{Z} ; \tau\right) W^{n} U^{m}
$$

we obtain that

$$
f\left(\theta_{Z} ; Z ; Y ; \tau\right) \preceq \sum_{n, m, r} \frac{(k+n+r) !(m+l) !}{(k+n+r+m+l+1) !} \bar{a}_{n m}\left(\theta_{Z}\right) Z^{n+r} Y^{m+r},
$$

where $\bar{a}_{n m}\left(\theta_{Z}\right)$ dominates $a_{n m}\left(\theta_{Z} ; \tau\right)$ over $\tau$ and $\preceq$ implies that $f\left(\theta_{Z} ; Z ; Y ; \tau\right)$ is dominated by the r.h.s. of (6.4). As anticipated, due to the exponential $\exp i \tau Z_{A} Y^{A}$ in (4.12), the expansion of the $f\left(\theta_{Z} ; Z ; Y ; \tau\right)$ is infinite even for polynomial $\phi^{\prime}(W ; U ; \tau)$. The expansion coefficients on the r.h.s. of (6.4) decrease faster for higher $k$ and $l$.

Note that solutions of differential equations always have a nonlocal form in terms of initial data with the nonlocality represented by the Green functions. Degree of nonlocality depends on the type of differential equations. Inclusion of higher derivatives makes the Green functions more nonlocal. The results of this paper suggest that the degree of nonlocality increases if the interaction terms contain central elements that do not belong to $\mathcal{H}$.

The algebra $\mathcal{H}^{l o c}$ is more restrictive than $\mathcal{H}$. The condition that the composition of any two local transformations has to be local is quite strong. From this perspective the fact that $\mathcal{H}^{l o c}$ forms an algebra is of crucial significance.

The proposed characterization of locality is justified by the fact that the HS equations reconstruct a solution in the class $\mathcal{H}$. This implies that by a shift of the HS fields valued 
in $\mathcal{H}$ it is possible to remove the r.h.s. of (3.3), i.e., it is cohomologically trivial in $\mathcal{H}$. Such a shift is analogous to a shift of a spin-one or spin-two field removing the current or stress tensor from the r.h.s. of the Maxwell or Einstein equations which is essentially nonlocal containing the Green function in the standard space-time picture. On the other hand, the r.h.s. of (3.3) cannot be removed by a field redefinition from $\mathcal{H}^{l o c}$.

The integrating flow of [1], leading to a nonlocal transformation removing the currents from the r.h.s. of the $3 d$ HS equations, is in fact strongly nonlocal, mapping the HS fields to $\mathcal{H}^{\text {tot }}$ as one can see from explicit formulae in $[1,11]$. Hence, as anticipated, it describes a nonlocal transformation in the setup of this paper.

General field redefinitions (6.1) can affect the form of the HS equations. Gauge transformations preserve the form of the HS equations. From the locality perspective it is important to distinguish between allowed and not allowed gauge transformations. The conjecture is that gauge transformations (3.9) with the gauge parameters valued in $\mathcal{H}$ are allowed. This is necessary, in particular, to make the perturbative analysis uniquely defined, allowing to gauge fix to zero the gauge freedom in $\varepsilon \in \mathcal{H}$ in (3.20). The gauge transformations with gauge parameters beyond $\mathcal{H}$, belonging for instance to $\mathcal{H}^{\text {tot }}$, cannot not be regarded as allowed gauge transformations since the transformed fields do not belong to $\mathcal{H}$.

The specification of the class of allowed gauge transformation is one of the most significant conclusions of this paper. This is important in particular in the context of application of quasi gauge transformations that "gauge away" the space-time dependence as, e.g., in $[10,13]$. Applying this trick one has to make sure that the final answer belongs to $\mathcal{H}$.

A subtlety in the analysis of locality is that proper prescription of the HS dynamics demands that physical fields $\phi^{\text {phys }}$ (see section 3.2) are identified with those in the $\mathrm{d}_{Z}$ cohomology in all orders of the perturbation theory

$$
\phi^{\text {phys }}=\phi_{1}^{\text {phys }}+\phi_{2}^{\text {phys }}+\phi_{3}^{\text {phys }}+\ldots,
$$

where $\phi_{n}$ describes a possible order- $n$ contribution. In other words, for a proper interpretation of a solution of the HS equations with HS fields valued in $\mathcal{H}$ one has to check that their reduction to the $\mathrm{d}_{Z}$ cohomology does not acquire nonlinear corrections in higher orders of the perturbative expansion. The canonical evaluation of perturbative corrections based on the application of the homotopy operator $\partial^{*}$ automatically obeys this condition since higher-order corrections do not contribute to the $\mathrm{d}_{Z}$ cohomology. Hence, the conjecture is that a proper solution to the HS equations should be locally equivalent to some canonical solution, i.e., equivalent up to transformations (6.1) from $\mathcal{H}^{l o c}$ or gauge transformations from $\mathcal{H}$.

\section{Conclusion}

Results of this paper are anticipated to shed light on the long-standing issue of locality in HS theories via the conjecture that local field redefinitions are from the class $\mathcal{H}^{l o c}$ while the gauge transformations are from the class $\mathcal{H}$. On the other hand, field redefinitions beyond the class $\mathcal{H}^{l o c}$ and gauge transformations beyond the class $\mathcal{H}$ should be regarded as 
nonlocal. In agreement with the expectation of [1], this conjecture rules out the pseudolocal field redefinitions resulting from the integrating flow of [1]. It would be interesting to test the locality conjecture by its application to other problems including the following.

The BH solutions of [14-16] need further investigation to clarify whether they belong to the proper class of functions. A closely related problem is that the solution of [14] was obtained in a nonstandard gauge that complicates its physical interpretation. From the perspective of this paper the problem is to bring the available solution to the class $\mathcal{H}$ by a formal gauge transformation. The analysis can also be affected by the fact that the behavior of the $\mathrm{BH}$ solutions in the $Y$ variables is essentially nonpolynomial, containing certain Fock vacuum factors [14]. This may require further specification of the proper functional classes in the $Y$ variables that can affect the issue of locality. From this perspective, being instrumental, consideration of this paper is not complete. Further investigation in spirit of, e.g., [17] (and references therein) would be desirable to elaborate appropriate restrictions on the coefficients $a_{n m}$ in (6.3) beyond the class of polynomials.

Analysis of this paper applies to the vacuum solutions that start with $Q(3.12)$ and to the homotopy operator of the form (3.21). As such, it is fully appropriate for the twistorial HS theories of $[1,3]$ but is less straightforward for other HS models. In particular, the construction of the vectorial HS models in any dimension of [4] involves the $s p(2)$ generators $\tau_{i j}$ which restrict the field pattern to the algebra $S$ of $s p(2)$ singlets followed by quotiening the ideal $I$ spanned by elements of the form $\tau_{i j} * f^{i j}$ or $f^{i j} * \tau_{i j}$. To apply the consideration of this paper to the vectorial models of [4] it is first of all necessary to check whether $\tau_{i j} \in \mathcal{H}$. This is likely to be true since $\tau_{i j}$ starts with elements of $\mathcal{H}$ bilinear in $Y$ in the lowest order and is reconstructed in higher orders by the equations analogous to the HS equations (3.4) (for more detail see [4, 18]). The next step is to choose representatives in $S / I$. A proper definition should be such that $S / I \in \mathcal{H}$. Practically, the choice of proper representatives of $S / I \in \mathcal{H}$ may be a tricky part of the analysis of the vectorial $H S$ theory.

The suggested setup helps to uncover the origin of the form of the nonlinear HS equations as encoding certain cohomology. This interpretation is useful for the search of more general models and approaches. For instance, in the lowest order of the perturbative expansion, the r.h.s. of the HS equations is valued in the boundary part of $\mathcal{H} / \mathcal{H}^{l o c}$, i.e., that at the boundary values of the homotopy parameter $\tau=0$ or 1 (see section 4.4). This property is anticipated to be true for other HS theories including those considered in [7], hence clarifying their structure. A surprising output of this paper is that certain central elements of the HS algebra, that naively look harmless, are ruled out from possible extensions of the r.h.s. of (3.3) as not belonging to the HS field algebra $\mathcal{H}$. In particular, the terms proportional to $\theta_{A} \theta^{A}$ are not allowed to appear anywhere in the HS equations except for the first term of (3.3). This is related to another unexpected conclusion that the $Z$-derivative is an outer derivation of the HS field algebra $\mathcal{H}$ because $Q$ (3.12) does not belong to $\mathcal{H}$. The condition that central elements that do not belong to $\mathcal{H}$ should not be allowed in the extended HS equations significantly restricts the space of extended HS systems and possible invariants among, for instance, those considered in [7, 9].

Analysis of this paper suggests the following interesting option. Consider the trivially 
looking system

$$
\begin{aligned}
\mathcal{W} * \mathcal{W} & =\mathcal{B}, \\
{[\mathcal{W}, \mathcal{B}]_{*} } & =0
\end{aligned}
$$

invariant under the gauge transformations

$$
\begin{aligned}
\delta \mathcal{W} & =\xi+[\mathcal{W}, \varepsilon]_{*}, \\
\delta \mathcal{B} & =\{\mathcal{W}, \xi\}_{*}+[\mathcal{B}, \varepsilon]_{*},
\end{aligned}
$$

where the gauge parameters $\varepsilon$ and $\xi$ are differential forms of even and odd degrees, respectively. This system is empty if $\mathcal{W}$ can be entirely gauge fixed to zero by the $\xi$ transformation.

Suppose however that

$$
\mathcal{W}, \mathcal{B}, \varepsilon \in \mathcal{H}, \quad \xi \in \mathcal{H}^{l o c}
$$

which assignment just fits the locality conjecture because the $\varepsilon$ transformation is the HS gauge transformation while the $\xi$ transformation shifting the fields $\mathcal{W}$ can in particular describe their nonlinear field redefinitions argued to belong to $\mathcal{H}^{l o c}$ in section 6 .

Eq. (7.2) then implies that $\mathcal{B}$ is perturbatively $Q$-closed. On the other hand, the first term in gauge transformation (7.4) washes out the $Q$-exact part of $\mathcal{B}$. As a result, upon gauge fixing the $\xi$-symmetry, the remaining components of $\mathcal{B}$ are in $\mathcal{H} / \mathcal{H}^{\text {loc }}$. Although the remaining part of equation (7.1) looks analogous to HS equations (3.3), there is an essential difference because so defined $\mathcal{B}$ is valued in the full cohomology $\mathcal{H} / \mathcal{H}^{l o c}$ rather than in the boundary cohomology with $\tau=0$ or 1 as the r.h.s. of (3.3).

As explained in section 4.4 , the full cohomology $\mathcal{H} / \mathcal{H}^{l o c}$ is represented by functions of $2 M$ variables. This space is far larger than the boundary cohomology represented by functions of a single variable $Y$ at $\tau=0$ or $Z$ at $\tau=1$. This makes it tempting to speculate that system (7.1)-(7.5) contains enough auxiliary degrees of freedom to represent the $3 d$ and $4 d$ off-shell HS systems. There is a subtlety however that it is not obvious whether this system respects the usual local Lorentz symmetry. We leave this problem for the future.

\section{Acknowledgments}

I am grateful to Vyatcheslav Didenko, Carlo Iazeolla, Nikita Misuna, Mikhail Soloviev and, especially, Olga Gelfond for useful comments and discussions. This research was supported by the Russian Science Foundation Grant No 14-42-00047.

\section{A Proof of Lemma 5}

Let $f\left(\theta_{Z} ; Z ; Y\right) \in V_{k, l, p}$. Changing the integration variables $(s, \tau)$ to $(s, t), t=s \tau$ and using the substitution (6.2), (4.44) gives

$$
\partial_{Z}^{*} f\left(\theta_{Z} ; Z ; Y\right)=\int \frac{d s}{s^{2}} d t \vartheta(t) \vartheta(s-t) \vartheta(1-s)(1-\tau)^{l} \tau^{k} Z^{A} \frac{\partial}{\partial \theta^{A}} \phi^{\prime}\left(s \theta_{Z} ; t Z ;(1-\tau) Y ; \tau\right) \exp i t Z_{A} Y^{A} \text {. }
$$


Let us focus on the case with regular dependence on $1-\tau$. (Distributions can be considered separately.) Since $\tau \geq t$ and the dependence on $1-\tau$ is demanded to be regular, all factors of $1-\tau$ are dominated by $1-t$. For $\phi^{\prime}\left(\theta_{Z} ; W ; U ; \tau\right)$ smooth in $\tau$ this gives

$$
\begin{aligned}
\partial_{Z}^{*} f\left(\theta_{Z} ; Z ; Y\right) \preceq \int d s d t \vartheta(t) \vartheta(s-t) \vartheta(1-s)(1-t)^{l} t^{k} s^{p-k-2} \\
Z^{A} \frac{\partial}{\partial \theta^{A}} \phi_{\max }^{\prime}\left(\theta_{Z} ; t Z ;(1-t) Y\right) \exp i t Z_{A} Y^{A},
\end{aligned}
$$

where $\preceq$ implies that the behavior on $\tau$ is dominated by the r.h.s. where $\phi_{\max }^{\prime}\left(\theta_{Z} ; W ; U\right)$ is some function regular in its arguments.

Integration over $s$ gives the factor of $\left(1-t^{p-k-1}\right)$. For $p>k+1$ it is dominated by $1-t$, i.e.,

$$
\partial_{Z}^{*} f\left(\theta_{Z} ; Z ; Y\right) \preceq \int_{0}^{1} d t(1-t)^{l+1} t^{k-1}\left(t Z^{A}\right) \frac{\partial}{\partial \theta^{A}} \phi_{\max }^{\prime}\left(\theta_{Z} ; t Z ;(1-t) Y\right) \exp i t Z_{A} Y^{A} .
$$

For $p<k+1,\left(1-t^{p-k-1}\right)$ is dominated by $t^{-p+k+1}(1-t)$ yielding

$$
\partial_{Z}^{*} f\left(\theta_{Z} ; Z ; Y\right) \preceq \int_{0}^{1} d t(1-t)^{l+1} t^{p-2}\left(t Z^{A}\right) \frac{\partial}{\partial \theta^{A}} \phi_{\max }^{\prime}\left(\theta_{Z} ; t Z ;(1-t) Y\right) \exp i t Z_{A} Y^{A} .
$$

In the case of $p=k+1$ the integration over $s$ develops a logarithmic dependence on $t$

$$
\partial_{Z}^{*} f\left(\theta_{Z} ; Z ; Y\right) \preceq \int_{0}^{1} d t(1-t)^{l} t^{k-1} \log (t)\left(t Z^{A}\right) \frac{\partial}{\partial \theta^{A}} \phi_{\max }^{\prime}\left(\theta_{Z} ; t Z ;(1-t) Y\right) \exp t Z_{A} Y^{A} .
$$

Since $\log (t)$ has a simple zero at $t \rightarrow 1$ and logarithmic singularities at $t \rightarrow 0$ do not affect indices of $V_{k, l, p}$ we finally obtain Lemma 5 .

\section{B Local HS algebra and Weyl star product}

Since polynomials of oscillators can be represented both in the Weyl (i.e., totally symmetric) and in the normal ordering prescriptions, the normal and Weyl star products of polynomials define the same algebra in different frames. The HS algebra is realized in terms of the particular normal-ordered star product (2.1). The intertwining relations between the Weyl and HS orderings are

$$
\begin{aligned}
f_{W}(Z ; Y) & =\frac{1}{(2 \pi)^{M}} \int d S d T \exp -i S_{A} T^{A} f_{H S}(Z+S ; Y+T), \\
f_{H S}(Z ; Y) & =\frac{1}{(2 \pi)^{M}} \int d S d T \exp i S_{A} T^{A} f_{W}(Z+S ; Y+T) .
\end{aligned}
$$

Indeed, it is not difficult to check that the star product $\star$ induced by substitution (B.2) from HS star product (2.1) yields the integral version of the Weyl-Moyal star product [5]

$$
\begin{aligned}
& \left(f_{W} \star g_{W}\right)(Z ; Y)= \\
& =\frac{1}{(2 \pi)^{2 M}} \int d U d V \exp \left[i\left(-U_{1 A} V_{1}^{A}+U_{2 A} V_{2}^{A}\right)\right] f_{W}\left(Z+U_{1} ; Y+U_{2}\right) g_{W}\left(Z+V_{1} ; Y+V_{2}\right) .
\end{aligned}
$$


Being equivalent for polynomials, different star products may be inequivalent beyond this class. This phenomenon has clear origin. Reordering of a monomial of any degree from one ordering prescription to another contributes to polynomials of lower degrees. For a nonpolynomial function, containing an infinite number of terms, the sum of the contributions to a particular (e.g., constant part) may diverge. Hence, beyond the class of polynomials, different star products may require more careful definition of the functional classes.

For $f$ (4.12), the map (B.1) yields

$$
\begin{aligned}
f_{W}(Z ; Y)= & \frac{1}{(2 \pi)^{M}} \int_{0}^{1} d \tau \int d S d T \exp \left[-i(1-\tau) S_{A} T^{A}+i \frac{\tau}{1-\tau} Z_{A} Y^{A}\right] \\
& \phi\left(\tau S+\frac{\tau}{1-\tau} Z ; Y+(1-\tau) T ; \tau\right) .
\end{aligned}
$$

Changing the variables $(1-\tau) T=U$ yields

$$
\begin{aligned}
f_{W}(Z ; Y)= & \frac{1}{(2 \pi)^{M}} \int_{0}^{1} d \tau(1-\tau)^{-M} \int d S d T \exp \left[-i S_{A} T^{A}+i \frac{\tau}{1-\tau} Z_{A} Y^{A}\right] \\
& \phi\left(\tau S+\frac{\tau}{1-\tau} Z ; Y+T ; \tau\right) .
\end{aligned}
$$

For $\phi(V ; U ; \tau)$ that behave as $(1-\tau)^{M-1+\epsilon}$ at $\tau \rightarrow 1$, the measure $d \tau(1-\tau)^{\epsilon-1}$ is well defined. However, the $Z$-dependent terms in the exponential and the argument of $\phi$ contain the factors of $(1-\tau)^{-1}$ divergent at $\tau \rightarrow 0$. As a result, the naive map from both $\mathcal{H}$ and $\mathcal{H}^{l o c}$ to the Weyl star product is ill defined. However, for $Z=0$ the map from $\mathcal{H}^{l o c}$ to the Weyl star product is well defined while that from $\mathcal{H}$ is not. This is in agreement with the fact that the supertrace is well defined in $\mathcal{H}^{l o c}$ but not in $\mathcal{H}$ (recall that, in accordance with (2.14), the supertrace in the Weyl star product is evaluated at $Z=0$ ).

Note that the more careful analysis of the relation between the two types of star product demands to take into account the equivalence relations (4.46) by choosing a representative in $\mathcal{H}^{l o c}$ obeying (may be appropriately modified) condition (4.48).

Open Access. This article is distributed under the terms of the Creative Commons Attribution License (CC-BY 4.0), which permits any use, distribution and reproduction in any medium, provided the original author(s) and source are credited.

\section{References}

[1] S.F. Prokushkin and M.A. Vasiliev, Higher spin gauge interactions for massive matter fields in 3 -D AdS space-time, Nucl. Phys. B 545 (1999) 385 [hep-th/9806236] [InSPIRE].

[2] S.F. Prokushkin and M.A. Vasiliev, Cohomology of arbitrary spin currents in $A d S_{3}$, Theor. Math. Phys. 123 (2000) 415 [hep-th/9907020] [INSPIRE].

[3] M.A. Vasiliev, More on equations of motion for interacting massless fields of all spins in (3+1)-dimensions, Phys. Lett. B 285 (1992) 225 [InSPIRE].

[4] M.A. Vasiliev, Nonlinear equations for symmetric massless higher spin fields in (A)dS(d), Phys. Lett. B 567 (2003) 139 [hep-th/0304049] [INSPIRE]. 
[5] F.A. Berezin and M.A. Shubin, Schrödinger Equation, Moscow University Press, Moscow Russia (1983).

[6] M.A. Vasiliev, Extended Higher Spin Superalgebras and Their Realizations in Terms of Quantum Operators, Fortsch. Phys. 36 (1988) 33 [INSPIRE].

[7] M.A. Vasiliev, Invariant Functionals in Higher-Spin Theory, arXiv:1504.07289 [INSPIRE]

[8] M.A. Vasiliev, On Conformal, SL $(4, \mathbb{R})$ and Sp $(8, R)$ Symmetries of $4 d$ Massless Fields, Nucl. Phys. B 793 (2008) 469 [arXiv:0707.1085] [INSPIRE].

[9] N. Boulanger and P. Sundell, An action principle for Vasiliev's four-dimensional higher-spin gravity, J. Phys. A 44 (2011) 495402 [arXiv: 1102.2219] [INSPIRE].

[10] M.A. Vasiliev, Algebraic aspects of the higher spin problem, Phys. Lett. B 257 (1991) 111 [INSPIRE].

[11] M.A. Vasiliev, Higher spin gauge theories: Star product and AdS space, hep-th/9910096 [INSPIRE].

[12] M.A. Vasiliev, Properties of equations of motion of interacting gauge fields of all spins in (3+1)-dimensions, Class. Quant. Grav. 8 (1991) 1387 [INSPIRE].

[13] S. Giombi and X. Yin, The Higher Spin/Vector Model Duality, J. Phys. A 46 (2013) 214003 [arXiv: 1208.4036] [INSPIRE].

[14] V.E. Didenko and M.A. Vasiliev, Static BPS black hole in 4d higher-spin gauge theory, Phys. Lett. B 682 (2009) 305 [arXiv: 0906. 3898] [InSPIRE].

[15] C. Iazeolla and P. Sundell, Families of exact solutions to Vasiliev's 4 D equations with spherical, cylindrical and biaxial symmetry, JHEP 12 (2011) 084 [arXiv:1107.1217] [INSPIRE].

[16] J. Bourdier and N. Drukker, On Classical Solutions of 4d Supersymmetric Higher Spin Theory, JHEP 04 (2015) 097 [arXiv: 1411.7037] [INSPIRE].

[17] M.A. Soloviev, Algebras with convergent star products and their representations in Hilbert spaces, J. Math. Phys. 54 (2013) 073517 [arXiv:1312.6571] [INSPIRE].

[18] X. Bekaert, S. Cnockaert, C. Iazeolla and M.A. Vasiliev, Nonlinear higher spin theories in various dimensions, hep-th/0503128 [INSPIRE]. 